Currently, NB medical students studying in French and enrolled in the Sherbrooke program split their 4-year degree between Sherbrooke and the University of Moncton and do their residencies at Dumont and other hospitals around the province.- Christine Morris, Fredericton

DOI:I0.1503/cmaj.060072

\section{Phase 2: Benchmarks to}

\section{accountability}

S etting wait times is not an end in itself, but a step toward a sustainable health care system, says the federal Wait Times Advisor.

Dr. Brian Postl said that instead of setting "hundreds and hundreds of benchmarks" we need to look at ways to make the first 5 benchmarks (CMAJ 2005;174:299), set in December, work. Postl addressed attendees at a Jan. I6 conference sponsored by the Canadian Health Coalition (CHC), a non-profit group that promotes medicare.

Sustainability depends on innovative operational improvements that will ensure appropriateness of care and consistent management, said Postl, president and CEO of the Winnipeg Regional Health Authority. Physician buy-in is also essential.

Postl's report on phase 2 of the federal wait times project, which will stipulate elements of system transformation, is due this spring.

In broad terms, Phase 2 will include engaging researchers to tie evidence to benchmarks, adopting new business practices, such as common wait lists for provinces or regions, employing wait-time coordinators so patients "know where they are," wait-time management training for health care professionals, and transparent waiting lists.

It will also outline the use of information technologies, including integration across health care systems and developing a registry.

Postl said physicians must also "take responsibility and change how they do business" by moving from a physician-based model to a program service-based culture. In the former, physicians "feel ownership of their patients" and refer them to, for example, a specific neurologist. Postl advocates a system where patients are assigned to a neurological service.

"We need to establish professional responsibility and accountability if this is going to work," said Postl. "We need physicians as part of it; we can't do it without them."

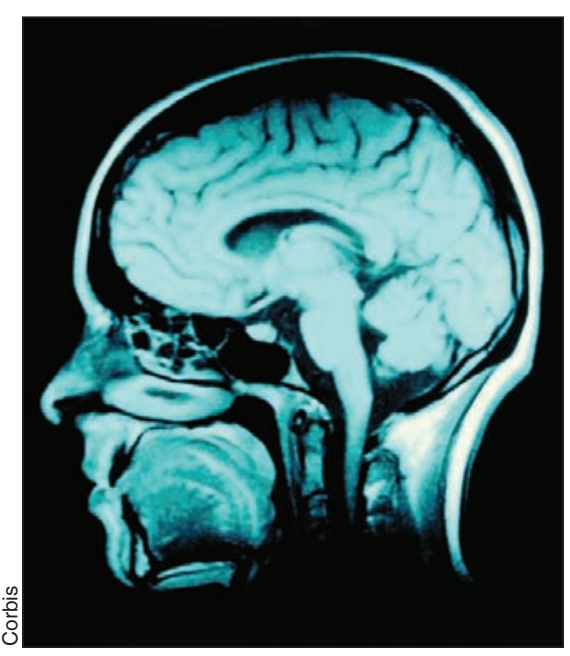

Using our head to reduce wait lists: about $5 \%$ of radiology imaging is unnecessary.

"One of the key issues around benchmarks and wait times, which we've done a very poor job of in this country, is the area of appropriateness," added Postl. There are some "remarkable inconsistencies across the country in how that's managed."

A case in point is the use of radiology services. A pilot study by the Canadian Association of Radiologists (CAR) found that $86 \%$ of radiology requests were appropriate, but in $9 \%$ of cases a more appropriate test should have been ordered and in $5 \%$ of cases no imaging was necessary for diagnosis or treatment.

This means that 4 million exams per year could be eliminated, saving about $\$ 500$ million or twice the annual equipment purchasing budget. This saving would mean the "elimination of wait times" and "the elimination of the need for private services," CAR's CEO Normand Laberge, told the conference. "We need to make sure we only do tests that will have a benefit."

Although these strategies are obviously needed, the problem is one of public perception, CHC Chair Kathleen Connors said at the conference. "The care guarantee has become the Trojan horse for for-profit health care." In an attempt to meet care guarantees, provinces will allow more privately delivered care, she maintained. - Barbara Sibbald, CMAJ

DOI:Io.I503/cmaj.o6oro6

\section{Quebec strain of C. difficile}

\section{in 7 provinces}

\section{$\mathrm{T}$} he same strain of Clostridium difficile that has caused close to I400 deaths in Quebec since 2003 is present in 7 provinces, the Public Health Agency of Canada is reporting.

The NAP I strain of $C$. difficile was found in hospitals in Ontario, Quebec, Nova Scotia, Newfoundland and Labrador, Alberta, Saskatchewan and British Columbia. The Canadian Nosocomial Surveillance Program participated in a 6-month study conducted by the Canadian Hospital Epidemiology Association and the Public Health Agency, and involving 34 hospitals that belong to the program (Fig. I).

From Nov. I, 2004, to Apr. 30, 2005, the National Microbiology Laboratory in Winnipeg collected more than 2000 stool samples and epidemiological data from patients at these institutions. The Public Health Agency has so far analyzed 615 of those samples and identified I847 cases of NAP I. Although they have not yet found the strain in New Brunswick and Manitoba, that may be because they still have more samples to investigate, says Denise Gravel, manager of the Nosocomial and Occupational Infections section of the Public Health Agency.

Compared to Agency data from a similar 1997 study, the incidence rate of $C$. difficile is essentially unchanged; $5.8 \%$ per 1000 hospital admissions in I997, and 6\% in the new study. But the 